\title{
A Modular Method for the Efficient Calculation of Ballistic Transport Through Quantum Billiards
}

\author{
S. Rotter, B. Weingartner, F. Libisch, F. Aigner, J. Feist, and J. Burgdörfer \\ Institute for Theoretical Physics, Vienna University of Technology, \\ A-1040 Vienna, Austria \\ stefan.rotter@tuwien.ac.at
}

\begin{abstract}
We present a numerical method which allows to efficiently calculate quantum transport through phase-coherent scattering structures, so-called "quantum billiards". Our approach consists of an extension of the commonly used Recursive Green's Function Method (RGM), which proceeds by a discretization of the scattering geometry on a lattice with nearest-neighbour coupling. We show that the efficiency of the RGM can be enhanced considerably by choosing symmetry-adapted grids reflecting the shape of the billiard. Combining modules with different grid structure to assemble the entire scattering geometry allows to treat the quantum scattering problem of a large class of systems very efficiently. We will illustrate the computational challenges involved in the calculations and present results that have been obtained with our method.
\end{abstract}

\section{Introduction}

A major aim in ballistic transport theory is to simulate and stimulate experiments in the field of phase-coherent scattering through nano-scaled semiconductor devices 7, 4. However, even for two-dimensional quantum dots ("quantum billiards") the numerical solution of the Schrödinger equation in an effective oneelectron approximation has remained a computational challenge. This is partly due to the fact that many of the most interesting phenomena occur in parameter regimes which are difficult to handle from a computational point of view: (1) In the "semi-classical regime" of high Fermi energy $E_{F}$ the de Broglie-wavelength of the electrons, $\lambda_{D}=2 \pi / \sqrt{2 E_{F}}$, is much smaller than the linear dimensions of the scattering device, $\lambda_{D} \ll D$. To properly describe the continuum limit of the transport process, a large number of basis functions is necessary [14]. Eventually this requirement renders all available methods computationally unfeasible or numerically instable. (2) In the "quantum-Hall regime" of very high magnetic fields the magnetic length, $l_{B}=\sqrt{c / B}$ (in atomic units), is considerably smaller than the system dimensions, $l_{B} \ll D$. Methods based on the expansion in plane or spherical waves become invalid since diamagnetic contributions are generally neglected 14. Methods employing a discretization on a grid do not allow the flux per unit cell to exceed a critical value and are therefore limited in the range of magnetic fields accessible [4,2. In this article we discuss a modification of the widely used Recursive Green's Function Method (RGM) 4] and illustrate 
how this modular extension of the RGM can bypass several of the limitations of conventional techniques.

\section{Method}

We consider a two-dimensional scattering geometry ("billiard") to which two semi-infinite waveguides ("leads") of width $d$ are attached in different orientations. A constant flux of electrons is injected at the Fermi energy $E_{F}=$ $\hbar^{2} k_{F}^{2} /\left(2 m_{\text {eff }}\right)$ through one of the waveguides and can leave the cavity through either the entrance or the exit lead. We assume inelastic scattering processes to be absent, such that the electronic motion throughout the device region is ballistic and therefore determined by the shape of the billiard. The potential surface inside the boundary of the dot is allowed to have different shapes (flat, soft wall profile or disordered) and is infinitely high outside. Atomic units $\left(\hbar=|e|=m_{\mathrm{eff}}=1\right)$ will be used, unless explicitly stated otherwise.

Our starting point is the standard recursive Green's function method (RGM). This approach is widely used in various fields of computational physics and consists in a discretization of the scattering geometry on a Cartesian grid. Setting up a tight-binding (tb) Hamiltonian on this grid,

$$
\hat{H}^{\mathrm{tb}}=\sum_{i} \varepsilon_{i}|i\rangle\left\langle i\left|+\sum_{i, j} V_{i, j}\right| i\right\rangle\langle j|,
$$

the hopping potentials $V_{i, j}$ and the site energies $\varepsilon_{i}$ are chosen such that the equation $\hat{H}^{\mathrm{tb}}\left|\psi_{m}\right\rangle=E_{m}\left|\psi_{m}\right\rangle$ converges towards the continuum one-particle Schrödinger equation, $[-\Delta / 2+V(x, y)]\left|\psi_{m}\right\rangle=E_{m}\left|\psi_{m}\right\rangle$, in the limit of high grid density. The hopping potentials are non-zero only for nearest-neighbour coupling of grid-points (with spacing $\Delta x, \Delta y$ ) and result directly from a three-point difference approximation of the kinetic energy term in the free-particle Hamiltonian [4,

$$
\varepsilon_{i}=\frac{1}{\Delta x^{2}}+\frac{1}{\Delta y^{2}}, \quad V_{i, i \pm 1}^{x}=\frac{-1}{2 \Delta x^{2}}, \quad V_{j, j \pm 1}^{y}=\frac{-1}{2 \Delta y^{2}} .
$$

With the help of the eigenvectors $\left|\psi_{m}\right\rangle$ and the eigenvalues $E_{m}$ of the Hamiltonian $\hat{H}^{\text {tb }}$ the Green's functions of one-dimensional tb strips are calculated,

$$
G^{ \pm}\left(\boldsymbol{x}, \boldsymbol{x}^{\prime}, E\right)=\lim _{\epsilon \rightarrow 0} \sum_{m} \frac{\left\langle\boldsymbol{x} \mid \psi_{m}\right\rangle\left\langle\psi_{m} \mid \boldsymbol{x}^{\prime}\right\rangle}{E \pm i \epsilon-E_{m}} .
$$

The different signs $( \pm)$ denote the retarded and advanced Green's functions, respectively. The disconnected transverse tb-strips incorporate the boundary conditions at the top and the bottom (see Fig. 13) as accurately as possible. The Green's functions of the strips are connected one at a time through recursive solutions of a matrix Dyson equation,

$$
G=G^{0}+G^{0} V G
$$


where $V$ is the hopping potential between the strips, $G^{0}$ and $G$ denote the retarded Green's function matrices of the disconnected and the connected tb strips, respectively. The complete scattering structure can thus be assembled from the individual strips much like knotting a carpet. Note that in this procedure the number of transverse strips is equal to the number of recursions (each involving at least one matrix inversion). For very high electron energies $E_{F}$, the large number of strips required to simulate the continuum eventually renders transport calculations impractical. This is in part because of the very large size of the matrices which have to be inverted in the strip-by-strip recursion process.

The remedy which we have proposed [8, 9, to overcome such difficulties goes back to Sols et al. [11] and consists of an extension of the RGM. Starting point is the observation that the efficiency of the "conventional" discretization employed in the RGM can be increased considerably by taking the symmetry of the scattering problem into account. More specifically, if the two-dimensional nonseparable open quantum dot can be built up from simpler separable substructures ("modules"), one gains significantly in computational speed by calculating the Green's functions for each of these modules separately. We solve the tight-binding Schrödinger equation, $\hat{H}^{\mathrm{tb}}\left|\psi_{m}\right\rangle=E_{m}\left|\psi_{m}\right\rangle$, now for one module at a time. Employing symmetry-adapted tight-binding grids leads to the separability of the eigenfunctions $\left|\psi_{m}\right\rangle$ for the modules and allows to determine the Green's function for an entire module [according to Eq. (3)] fast and virtually exactly. For joining modules with each other we employ the technique of the RGM where the coupling between Green's functions is facilitated in terms of the corresponding hopping matrix elements of the tight-binding Hamiltonian. By solving one Dyson equation at each junction between the modules, the complete scattering structure can be assembled much like a jigsaw puzzle. In Fig. 10 we illustrate how the discretization of the circle billiard within the framework of this modular recursive Green's function method (MRGM) 8, 9] proceeds.

Note that, quite in contrast to the conventional RGM, the number of recursions (i.e., of matrix inversions) needed to obtain the Green's function of the total scattering problem is given by the number of separate modules required to build up the scattering structure. This number is independent of the de Broglie wavelength. The latter enters only in terms of the size of the matrices involved in the fixed number of recursions. Furthermore, for solving the transport problem, the module Green's functions have to be evaluated only on the subset of grid points which are coupled to grid points on neighbouring modules. Another advantage of the MRGM comes into play when solving the scattering problem at different Fermi energies $E_{F}$, since for all values of $E_{F}$ the eigenvalue problem Eq. (1) has to be solved only once for each module. This is because the eigenvectors $\left|E_{m}\right\rangle$ and the eigenenergies $E_{m}$ are independent of $E_{F}$. As a consequence of these advantages it is possible to incorporate a very high number of grid points in the calculations, which is the prerequisite to access the "semiclassical" as well as the "quantum Hall" regime. On the negative side, the most severe restriction of the MRGM is its restricted applicability to those scattering structures which can be assembled from separable modules. This includes random and soft-wall 


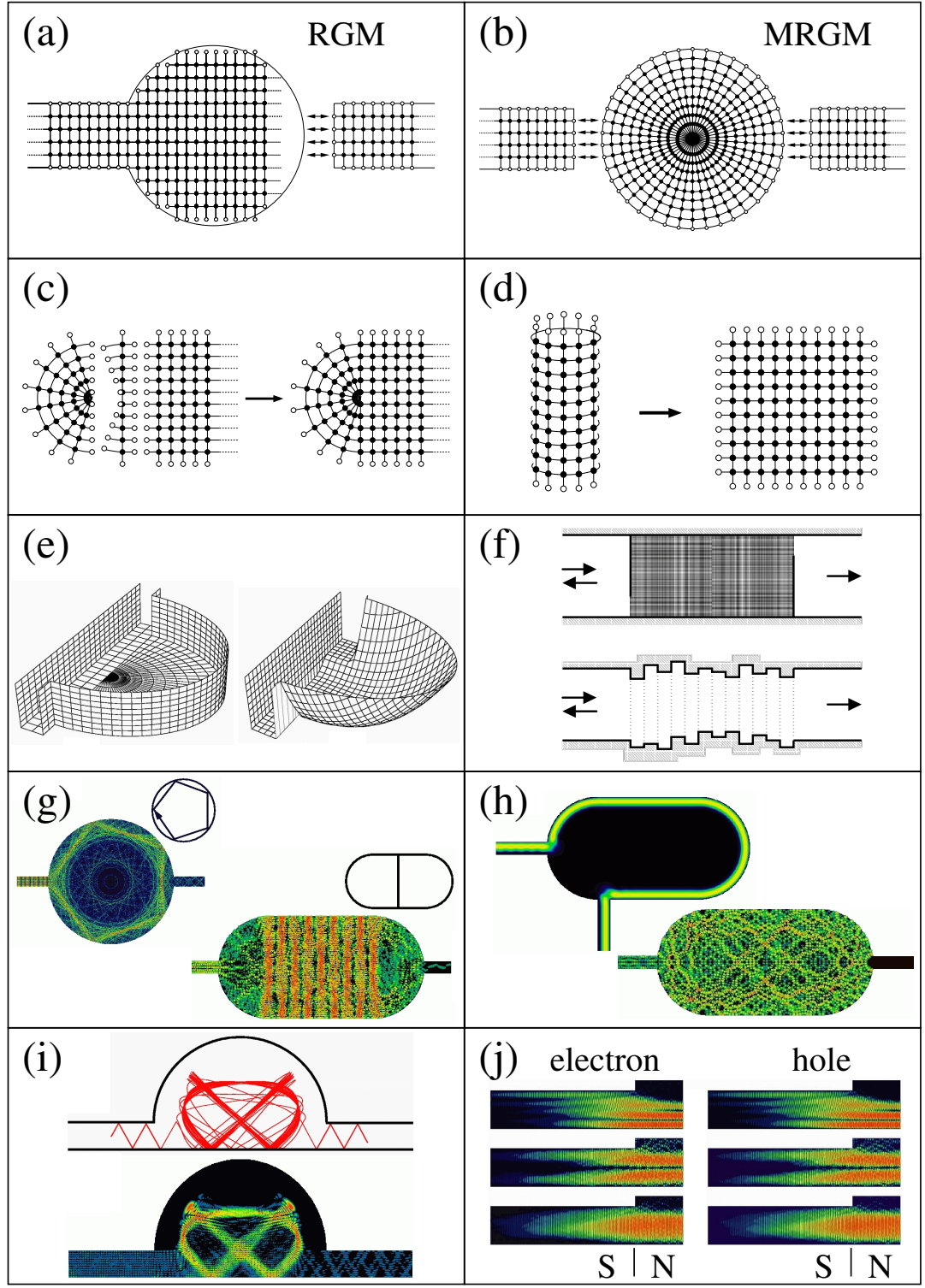

Fig. 1. (Color) Discretization of the circular billiard with leads employing (a) the conventional RGM and (b) the MRGM. (c)-(d) Features of the discretization with the MRGM (see text for details). (e) Hard wall vs. soft wall profile. (f) Bulk and surface disorder potentials. (g) Density of localized wavefunctions $|\psi(x, y)|^{2}$ in a regular vs. chaotic billiard. (h) Density of scattering wavefunctions in the high magnetic field vs. high energy limit. (i) "Trapped" trajectory in a soft wall billiard with a mixed classical phase space and the density of the corresponding quantum wavefunction. (j) Three bound electron-hole wavefunction densities in a square billiard with superconducting lead ("Andreev billiard"). 
potentials as long as they preserve the separability of each module. For problems which do not allow for a decomposition into modules, other variants of standard lattice Green's function methods could be employed [6, 15].

Once the Green's function $G\left(\boldsymbol{x}, \boldsymbol{x}^{\prime}, E\right)$ for the total scattering geometry is determined, the scattering wave functions can be obtained by projecting this Green's function onto the transverse states $\chi_{n}(y)=(2 / d)^{1 / 2} \sin (n \pi y / d)$ in the entrance lead,

$$
\psi_{m}\left(\boldsymbol{x}, E_{F}\right)=i \sqrt{k_{x, m}} \int_{-d / 2}^{d / 2} d y^{\prime} G\left(\boldsymbol{x}, \boldsymbol{x}^{\prime}, E_{F}\right) \chi_{m}\left(y^{\prime}\right) .
$$

The amplitudes $t_{n m}$ for transmission from the entrance lead mode $m$ to exit lead mode $n$ can be calculated by projecting the scattering wavefunction $\psi_{m}\left(\boldsymbol{x}, E_{F}\right)$ onto the transverse wavefunction in the exit lead $\chi_{n}\left(y_{i}\right)$,

$$
t_{n m}\left(E_{F}\right)=-i \sqrt{k_{x, n} k_{x, m}} \int_{-d / 2}^{d / 2} d y \int_{-d / 2}^{d / 2} d y^{\prime} \chi_{n}^{*}(y) G\left(\boldsymbol{x}, \boldsymbol{x}^{\prime}, E_{F}\right) \chi_{m}\left(y^{\prime}\right),
$$

where $k_{x, n}$ denotes the corresponding longitudinal wave numbers, $k_{x, n}=\left[k_{F}^{2}-\right.$ $\left.(n \pi / d)^{2}\right]^{1 / 2}$. The indices $n, m \in[1, \ldots, M]$, with $M$ denoting the total number of open channels in the leads. The wave numbers $k_{x, n}$ are real for $n \pi / d<k_{F}$ (open channels). For $n \pi / d>k_{F}$ (closed channels), $k_{x, n}$ is purely imaginary. According to the Landauer formula [4, the total conductance $g$ through the quantum dot is given by

$$
g=\frac{1}{\pi} \sum_{m, n=1}^{M}\left|t_{n m}\right|^{2}=\frac{1}{\pi} T^{\text {tot }},
$$

which is an experimental observable in semiconductor devices.

\section{$3 \quad$ Special Features and Sample Results}

\subsection{Joining Modules of Different Symmetry}

To investigate quantum billiards which feature chaotic classical dynamics it becomes necessary to turn to cavity geometries which are different from the purely separable cases. A prototypical example in this context is the stadium billiard (see e.g. Fig. 1h and [12), for which two half-circular and one rectangular module are joined with each other. Using the MRGM we are confronted with the problem of how to properly connect the Cartesian grid of the rectangle with the polar grid structure of the half-circles without violating the Hermiticity of the tight-binding Hamiltonian at the junctions. To overcome this problem we insert additional link modules between the rectangle and the half-circles (see Fig. 15). These link modules are essentially one-dimensional strips the site energies of which contain contributions from both adjacent grid structures [8].

In Fig. 18 we present two scattering wavefunctions for the circle and the stadium, respectively 9. Note how the two wavefunctions in Fig. 19 are both localized along a typical classical trajectory in the respective cavity. 


\subsection{Magnetic Field}

The MRGM allows to incorporate a magnetic field $\mathbf{B}$ oriented perpendicular to the 2D scattering geometry. The field enters the tb Hamiltonian of Eq. (1) by means of a Peierls phase factor [4,2, with which the field-free hopping potential $V_{i, j}$ is multiplied: $V_{i, j} \rightarrow V_{i, j} \times \exp \left[i / c \int \mathbf{A}(\mathbf{x}) \mathbf{d x}\right]$. The vector potential $\mathbf{A}(\mathbf{r})$ satisfies $\nabla \times \mathbf{A}(\mathbf{r})=\mathbf{B}$. The Peierls phase will, of course, in most cases destroy the separability of the eigenfunctions of $\hat{H}^{\mathrm{tb}}$ in the modules. The resulting difficulties can be, in part, circumvented by exploiting the gauge freedom of the vector potential, i.e., $\mathbf{A} \rightarrow \mathbf{A}^{\prime}=\mathbf{A}+\nabla \lambda$, where $\lambda(\mathbf{r})$ is a scalar function. By an appropriate choice of $\lambda$ the wavefunction may remain separable on a given symmetry-adapted grid. Note, however, that even in the seemingly simple case of a rectangular module separability is destroyed by the magnetic field, no matter which gauge is chosen. The separability can however be restored by imposing periodic boundary conditions on two opposing sides of the rectangle. Topologically, this corresponds to folding the rectangle to the surface of a cylinder. The Green's function for the rectangle is finally obtained from the cylinder Green's function by a Dyson equation which is used here in "reversed" mode, i.e. for disconnecting tb grids (see Fig. 11) 9 .

The top part of Fig. 1h shows a stadium wavefunction in the very high magnetic field limit 9 . The magnetic field leads to the emergence of so-called "edge states" which creep along the boundary of the billiard [4. The bottom part of Fig. 1h displays a field-free stadium wavefunction (in the high energy limit) which explores the entire cavity area.

\subsection{Soft Potential Walls}

Most theoretical investigations on quantum billiards focus on the two limiting cases of systems with either purely chaotic or purely regular classical dynamics [12. However, neither of these cases is generic. For the semiconductor quantum dots that are realized in the experiment [4] a classical phase space structure with mixed regions of chaotic and regular motion is expected. This is due to the fact that the boundaries of such devices are typically not hard walls (as assumed in most theoretical investigations) but feature soft wall profiles [4] for which such a "mixed" phase space is characteristic. Soft walls can be incorporated in the MRGM, as long as their potential profiles do not violate the separability condition within a given module. In Fig. 1 we show an example of how this requirement can be fulfilled in the particular case of a half-stadium geometry (left part: hard wall case, right part: soft walls). Classical simulations reveal that the half-stadium with smooth boundaries indeed features a phase space within which regular and chaotic motion coexists. Characteristic for such "mixed systems" are very long trajectories that get "trapped" in the vicinity of regular islands of motion [12. A typical example for such a trajectory is depicted in the top part of Fig. 11. The quantum scattering wavefunction which corresponds to this orbit is shown right below [13]. 


\subsection{Bulk and Surface Disorder}

For many fundamental quantum transport phenomena the presence of disorder is essential and determines whether transport will be ballistic, diffusive or entirely suppressed by localization [4. Whereas the (mostly unwanted) disorder is naturally present in the experiment, it is not straightforward to simulate disorder numerically. Bulk disorder can be viewed as random variations of the potential landscape through which the electron is transported. Within the framework of the MRGM the inclusion of such non-separable potential variations is not obvious, since separability is required in each of the modules. A way to overcome this difficulty is depicted in the top inset of Fig. 1f; where we decompose the cavity region into two square modules for each of which we choose a separable random potential $V(x, y)=V_{1}(x)+V_{2}(y)$. In order to destroy the unwanted separability, we combine two identical modules, however, rotated by $180^{\circ}$ relative to each other [1. The case of surface disorder can be treated by compiling a large number of rectangular modules of variable height (see bottom part of Fig. 1ff). In this context the amount of spatial variation of the transverse module widths represents the strength of the disorder.

Since diffusion and localization in transport occur on very extended spatial scales, it is necessary to build up disorder regions of large length. For this purpose we employ an approach which allows to "exponentiate" the iteration process [10]: in the first step of the Dyson iteration we join individual rectangular modules with each other and employ the resulting combination of modules as the building block for the next step of the iteration. Repeating this procedure in each step of the iteration process, we manage to increase the size of the disordered region exponentially — with only a linear increase of computational time [3].

\subsection{Andreev Billiards}

The interface between a normal-conducting $(\mathrm{N})$, ballistic quantum dot and a superconductor (S) gives rise to the coherent scattering of electrons into holes. This phenomenon is generally known as Andreev reflection. A N-S hybrid structure consisting of a superconducting lead attached to a normal cavity (see Fig. 1j) is commonly called an Andreev billiard. Such billiard systems attracted much attention recently, especially because of the unusual property that the classical dynamics in these systems features continuous families of periodic orbits, consisting of mutually retracing electron-hole trajectories. To learn more about the classical-to-quantum correspondence of Andreev billiards it is instructive to study the bound states in these billiards and the form of their wavefunctions. As shown in Fig. 1], such wavefunctions indeed feature an electron and a hole part that (in most cases) closely resemble each other - in analogy to the classical picture of retracing electron-hole orbits. To obtain these quantum results numerically, we calculated the scattering states for the billiard with a normal conducting lead and entangled them in a linear superposition to construct the Andreev states [5. Work on a more versatile approach is in progress which features coupled electron and hole tight-binding lattices explicitly. 


\section{Summary}

We have given an overview of the modular recursive Green's function method (MRGM), which allows to calculate ballistic transport through quantum billiards efficiently. Key feature of the MRGM is the decomposition of billiard geometries into separable substructures ("modules") which are joined by recursive solutions of a Dyson equation. Several technical aspects of the method, as well as computational challenges and a few illustrative results have been presented.

\section{Acknowledgments}

Support by the Austrian Science Foundation (FWF Grant No. SFB-016, FWFP17359, and FWF-P15025) is gratefully acknowledged.

\section{References}

1. Aigner, F., Rotter, S., and Burgdörfer, J.: Shot noise in the chaotic-to-regular crossover regime. Phys. Rev. Lett. 94 (2005) 216801

2. Baranger, H. U., DiVincenzo, D. P., Jalabert, R. A., Stone, A. D.: Classical and quantum ballistic-transport anomalies in microjunctions. Phys. Rev. B 44 (1991) 10637

3. Feist, J., Bäcker, A., Ketzmerick, R., Rotter, S., Huckestein, B., Burgdörfer, J. (to be published)

4. Ferry, D. K. and Goodnick, S. M.: Transport in Nanostructures. (Cambridge University Press, 1997) and references therein

5. Libisch, F., Rotter, S., Burgdörfer, J., Kormányos, A., and Cserti, J.: Bound states in Andreev billiards with soft walls. Phys. Rev. B 72 (2005) 075304

6. Mamaluy, D., Sabathil, M., and Vogl, P.: Efficient method for the calculation of ballistic quantum transport. J. Appl. Phys. 93 (2003) 4628

7. Marcus, C. M., Rimberg, A. J., Westervelt, R. M., Hopkins, P. F., and Gossard, A. C.: Conductance fluctuations and chaotic scattering in ballistic microstructures. Phys. Rev. Lett. 69 (1992) 506

8. Rotter, S., Tang, J.-Z., Wirtz, L., Trost, J., and Burgdörfer, J.: Modular recursive Green's function method for ballistic quantum transport. Phys. Rev. B 62 (2000) 1950

9. Rotter, S., Weingartner, B., Rohringer, N., and Burgdörfer, J.: Ballistic quantum transport at high energies and high magnetic fields. Phys. Rev. B 68 (2003) 165302

10. Skjånes, J., Hauge, E. H., and Schön, G.: Magnetotransport in a two-dimensional tight-binding model. Phys. Rev. B 50 (1994) 8636

11. Sols, F., Macucci, M., Ravaioli, U., and Hess, K.: Theory for a quantum modulated transistor. J. Appl. Phys. 66 (1989) 3892

12. Stöckmann, H.-J.: Quantum Chaos. (Cambridge University Press, 1999) and references therein

13. Weingartner, B., Rotter, S., and Burgdörfer, J.: Simulation of electron transport through a quantum dot with soft walls. Phys. Rev. B 72 (2005) 115342

14. Yang, X., Ishio, H., and Burgdörfer, J.: Statistics of magnetoconductance in ballistic cavities. Phys. Rev. B 52 (1995) 8219

15. Zozoulenko, I. V., Maaø, F. A., and Hauge, E. H.: Coherent magnetotransport in confined arrays of antidots. Phys. Rev. B 53 (1996) 7975 